\title{
Correlation between health literacy and health-related quality of life in patients with hypertension, in Tehran, Iran, 2015-2016
}

\author{
Ahmad Johari Naimi ${ }^{1}$, Nadereh Naderiravesh ${ }^{2}$, Zahra Safavi Bayat ${ }^{2}$, Nezhat Shakeri $^{3}$, Mahsa Matbouei ${ }^{4}$ \\ ${ }^{1}$ M.Sc. of Nursing, Department of Medical-Surgical Nursing, School of Nursing \& Midwifery, Shahid Beheshti \\ University of Medical Sciences, Tehran, Iran \\ ${ }^{2}$ Ph.D. of Nursing, Assistant Professor, Department of Medical-Surgical Nursing, School of Nursing \& Midwifery, \\ Shahid Beheshti University of Medical Sciences, Tehran, Iran \\ ${ }^{3}$ Ph.D. of Biostatistics, Asistant Professor, Department of Biostatistics, School of Paramedical Sciences, Shahid \\ Beheshti University of Medical Sciences, Tehran, Iran \\ ${ }^{4}$ M.Sc. of Nursing, Department of Community Health Nursing, School of Nursing \& Midwifery, Shahid Beheshti \\ University of Medical Sciences, Tehran, Iran
}

\section{Type of article: Original}

\begin{abstract}
Background and Objective: Hypertension is considered an important public health problem in developed and developing countries. This disease is closely associated with the quality of life of patients, and it seems that health literacy plays a role in this regard. Due to lack of information on the role of health literacy on the quality of life in patients with hypertension, this study has aimed to determine the correlation between health literacy and health-related quality of life in patients with hypertension.

Methods: This study is cross-sectional. The sample consisted of 400 patients with hypertension who were enrolled if available. To collect data, a demographic questionnaire (SF-36), Short Form Health Survey, and Health Literacy for Iranian Adults (HELIA) Inventory were used. Data were analyzed using SPSS software version 21 for descriptive and inferential statistics (e.g., mean, standard deviation, t-test, and Pearson correlation coefficient).

Results: The mean and standard deviation scores of health literacy and health-related quality of life were, respectively, $68.66( \pm 13.56)$ and $52.94( \pm 15.20)$. There was positive and significant correlation between health literacy and health-related quality of life $(\mathrm{p}<0.01, \mathrm{r}=0.30)$.

Conclusion: According to research findings, there was a significant positive correlation between health literacy and health-related quality of life. Given the importance of health literacy in the quality of life in patients with hypertension, it seems essential that nursing officials and policymakers take steps to promote patients' health and quality of life by using training programs appropriate for the patients' health literacy level.
\end{abstract}

Keywords: Health literacy, Quality of life, Hypertension

\section{Introduction}

In the twenty-first century, the most outstanding event that communities and health workers face is the prevalence of chronic disease (1). Hypertension is one of those diseases and is a major public health problem in developed countries and developing countries; it is referred to as the silent killer, and, according to the World Health Organization, after tobacco, high blood pressure has been introduced as the second main risk factor of death in developing countries (2). Research shows that, each year, this disease involves more than 1.5 billion people in the world, and about 7 million people a year die of the disease (3). Epidemiological studies in Iran show the prevalence

\section{Corresponding author:}

Assistant Professor Dr. Nadereh Naderiravesh, Department of Medical-Surgical Nursing, School of Nursing \& Midwifery, Shahid Beheshti University of Medical Sciences, Tehran, Iran.

Tel: +98.9123505189, Email: N.Naderiravesh@sbmu.ac.ir

Received: January 10, 2017, Accepted: June 02, 2017, Published: November 2017

iThenticate screening: June 01, 2017, English editing: October 12, 2017, Quality control: October 15, 2017

This article has been reviewed / commented by Three experts

(C) 2017 The Authors. This is an open access article under the terms of the Creative Commons Attribution-NonCommercialNoDerivs License, which permits use and distribution in any medium, provided the original work is properly cited, the use is non-commercial and no modifications or adaptations are made. 
of this disease in society, and a large number of hypertensive patients in Iran was unaware of the existence of this disease; further, unrecognized and treated cases do not have adequate and appropriate control over their disease (4). International experience suggests that factors such as economic development, education promotion, health literacy, and developed social services will have a positive impact on health and social welfare of the population (5). One of the factors is health literacy, which is a term introduced in 1970 (6). Health literacy is the individual capacity to obtain, process, and understand basic information and health services to decide appropriately. The capacity of a person is a basic element for health literacy, which depends both on the individual inherent potential and his skills. This capacity can be adjusted with training, and its adequacy is determined via health-related activities (7). A study conducted by Tehrani Bani Hashemi et al. found that low levels of health literacy in the elderly, immigrants, illiterates, low-income people, people with low mental health, and people with chronic diseases such as type II diabetes and hypertension are more common (8). On the one hand, considering the increasing number of patients with hypertension in Iran and the world as well as its adverse effects on quality of life, it is of utmost importance (9). Patients with hypertension are placed in a category of chronic patients, and they must take medication for a long time. Given the importance and impact of chronic diseases on the quality of life and its various aspects (physical and mental health and social welfare), the attention of nurses to the concept of quality of life is particularly important (10). Because, in most clinical trials, health-related quality of life has been used as an evaluation criteria, to achieve the treatment results (11). Quality of life is a logical and conceptual process on the basis of culture and a summary of the values, beliefs, symbols, and experiences shaped by that culture (12). Lin et al. suggest that, although the health-related quality of life and quality of life are used interchangeably, they have two different meanings: quality of life encompasses all aspects of life, as the health-related quality of life refers to the impact of the disease and treatment on quality of life (13). Lower health literacy is associated with lack of knowledge about hypertension, but, unlike previous research on the relationship between health literacy and disease outcomes in diabetes, heart failure, asthma, and AIDS, there was no strong and stable associated with health literacy and blood pressure control (14). As in research in the field of comprehensive disease management program, systolic blood pressure improved without change in patients' health literacy status (15). Another study also found that high levels of health literacy in patients is directly related to high blood pressure (16); on the other hand, the research showed that the relationship between health literacy of patients with hypertension and systolic blood pressure may vary by health service delivery models (17). In total, it is important that the awareness of health literacy is needed to achieve blood pressure control (14) But, given that this issue is not addressed in Iran, and there is no statistics and evidence of assessment of correlation between health literacy and health-related quality of life in patients with hypertension; thus, according to the abovementioned subjects, the researcher decided to conduct a study in order to determine the correlation of health literacy with health-related quality of life in patients with hypertension.

\section{Material and Methods}

\subsection{Research design and participants}

The present study was a cross-sectional study. The study population, consisting of 400 patients with hypertension, was estimated based on similar studies. Sampling method was two-stage. First among the seven hospitals affiliated with Shahid Beheshti University of Medical Sciences in Tehran with the heart clinic, randomly four hospitals (Shohada Tajrish hospital, Modares, Imam Hussein, and Taleghani) were selected as sample. Then, in the second stage available, samples referred to the selected hospitals that had inclusion criteria, i.e., primary or secondary hypertension diagnosis by a cardiologists, at least six months of hypertension and history of using a controller medication for hypertension, at least 18 years of age and residing in Tehran, the ability to speak, ability for reading and writing Persian texts, were selected. One hundred samples were collected from each hospital. Patients who did not have a desire to continue to participate in research or had cognitive problems were excluded.

\subsection{Instrument}

Data were collected by demographic questionnaire, (SF-36) Short Form Health Survey Questionnaire, and Health Literacy for Iranian Adults (HELIA) Inventory. In the demographic questionnaire, the variables such as age, sex, education, marital status, employment status, the means of obtaining information health and disease, and history of hypertension were collected. "Questionnaire (SF-36)" was designed by Warr et al. in America (18). The purpose of this questionnaire is to evaluate health-related quality of life, both physically and mentally, obtained by combining the scores of eight dimensions of the questionnaire. This questionnaire contains 36 questions in which eight items included public health (five questions, including questions 1, 33, 34, 35, 36), physical function (10 questions, including questions three to 12), role limitations due to physical reasons (four questions, including questions 13 and 16), role limitations due to emotional reasons (three questions, including questions 17 and 19), mental health (five questions, including questions of 24-25-26-28-30), bodily pain (two questions, including questions 21 and 22), 
fatigue or vitality (four questions, including questions of 23,27, 29, and 31), social function (two questions, including questions 20 and 32).

For the grading scale, Question 2 was not in any of the following subscales and is only added with the total score. Scoring method is determined according to the number of options of each item. For example, the first item of the questionnaire includes five options, at best condition, the score 100 and at worst the score of zero have been given and the option between the two have the scores 75,50 , and 25 . In general, the questionnaire is divided into two aspects of physical and mental health: physical health included subscales of mental health, role limitations for physical reasons, bodily pain, and physical functioning; mental health included subscales of role limitations due to emotional reasons, social functioning, fatigue or vitality, and mental health. The score of each dimension is calculated according to the score of its subscales. As the total score in each subscale are added and divided by the number of the subscale in each dimension and thus physical and psychological scores are calculated. To calculate the total score of the questionnaire, the sum of the values obtained from each subscale is divided by number 8 (the total number of subscales) that the number obtained must be a number between zero and 100 . The lowest score on this questionnaire is zero and the highest is 100 that, zero shows the worst and 100 shows the best condition in Moore's scale. According to Montazeri et al., analysis showed internal consistency that except vitality scale $(\alpha=0.65)$, other scales (SF-36) have the minimum standard of reliability coefficients in the range of $0.77-0.90$. The convergent validity test provides good results to investigate measurement hypotheses using the correlation of each question or the scale hypothesized, and all correlation coefficients are obtained greater than the recommended value of 0.4 (19). Test-retest reliability of 0.75 for this questionnaire with an interval of two weeks is obtained in Dehdari et al.'s study (20). "The urban population's health literacy questionnaire" was designed by Montazeri et al. The tool consists of 33 questions in five domains that the domain of access, understanding, evaluation, and decision-making, respectively, contain six, seven, four, and 12 items that the answers "always," "most of the time," "sometimes," and "never" to question assigned the scores 5, 4, 3,2, and 1, respectively, and the area of reading skill consists of four questions, the answers "quite easy," "easy," "neither easy nor hard," "hard," and quite hard assigned the scores 5, 4, 3,2 , and 1 , respectively. In the urban population's health literacy questionnaire, minimum and maximum scores on a Likert scale of 1 to 5, were, respectively, 33 and 165. Calculation of total health literacy score in a Likert scale was obtained by dividing these scores by 33 (total number of questions) that a number between 1 and 5 was obtained. According to the division of Likert questions, answers $<3$ were considered as inadequate, 3-4 as boundary and $>4$ were considered as sufficient. To indicate the numbers on a scale of 0 to 100 , all numbers obtained on the Likert scale (1-5) were transferred to a 100-point scale. Thus, the answers $<50$ were considered as inadequate, 50-75 as boundary, and $75.1<$ were classified as adequate. The questionnaire with 33 questions on 5 areas had good construct validity and Cronbach's alpha of the items in the relevant structures is acceptable ( 0.72 to 0.89 and is approved in terms of the reliability of the questionnaire (21).

\subsection{Data collection, ethics, and analysis}

The researcher, after obtaining the introduction letter from the Shahid Beheshti Nursing and Midwifery Department and research license from the university ethics committee No. IR.SBMU.PHNM.1394.156, referred to cardiology clinics of the selected hospitals. After verification of hospitals' authorities, researchers while introducing themselves to the subjects, giving information about the research objectives, obtaining their consent, and completing an informed consent form, collected information. Data were analyzed by IBM $\odot$ SPSS $\odot$ Statistics version 21 (IBM $\odot$ Corp., Armonk, NY, USA) using descriptive and inferential statistics (mean, standard deviation, t-test, and Pearson correlation coefficient) at the significance level of 0.05 .

\section{Results}

Results showed that the mean age of patients with hypertension who participated in the study was $42.46( \pm 14.61)$ years and more in age group 40 to 49 years: $55 \%$ of participants were male and $66.5 \%$ were married; $36 \%$ of respondents had education higher than diploma; $32.7 \%$ were employed; $47.5 \%$ obtained information of health and disease through radio and television. The mean duration of hypertension in participants of this study was 4.10 ( \pm 3.66$)$ years and more participants $(78.8 \%$ ) have a history of hypertension for 1 to 5 years (Table 1$)$. The average score for quality of life in patients was $52.94( \pm 15.20)$; among the eight dimensions of health-related quality of life, the highest score of $70.12( \pm 24.55)$ was observed in physical function. Conducting paired t-test between the physical and mental health subscales, significant difference was observed $(\mathrm{p}=0.001)$. Thus, under the mental health subscale $54.21( \pm 17.14)$ assigned higher score in comparison to the subscales of mental health $51.68( \pm 17.07)$ (Table 2). According to Table 3, the mean score of health literacy was $68.66( \pm 13.56)$ of 100 . The domain of understanding with the score of $70.15( \pm 17.39)$ had the greatest and domain of decision-making with the score of $53.07( \pm 12.84)$ 
was lowest; $7.8 \%$ of patients with hypertension had inadequate health literacy; $55 \%$ were in boundary level; and $37.2 \%$ had adequate health literacy. Table 4 indicates the positive and significant correlation between health literacy and health-related quality of life.

Table 1. Frequency of distribution of demographic characteristics of patients

\begin{tabular}{|c|c|c|c|}
\hline \multicolumn{2}{|l|}{ Variable } & $\mathrm{n}$ & $\%$ \\
\hline \multirow[t]{2}{*}{ Sex } & Male & 220 & 55 \\
\hline & Female & 180 & 45 \\
\hline \multirow{8}{*}{ Age (year) } & $18-19$ & 7 & 1.8 \\
\hline & $20-29$ & 86 & 21.5 \\
\hline & $30-39$ & 83 & 20.7 \\
\hline & $40-49$ & 98 & 24.5 \\
\hline & $50-59$ & 72 & 18 \\
\hline & $60-69$ & 39 & 9.7 \\
\hline & $70-79$ & 7 & 1.8 \\
\hline & $80-89$ & 8 & 2 \\
\hline \multirow[t]{6}{*}{ Education } & reading and writing & 39 & 9.7 \\
\hline & Primary & 25 & 6.3 \\
\hline & Guidance & 41 & 10.2 \\
\hline & High school & 28 & 7 \\
\hline & Diploma & 123 & 30.8 \\
\hline & Higher & 144 & 36 \\
\hline \multirow[t]{4}{*}{ Marital status } & Single & 114 & 28.5 \\
\hline & Married & 266 & 66.5 \\
\hline & Divorced & 9 & 2.3 \\
\hline & Widow(er) & 11 & 2.7 \\
\hline \multirow[t]{7}{*}{ Employment status } & Unemployed & 8 & 2 \\
\hline & housewife & 75 & 18.8 \\
\hline & Retired & 58 & 14.5 \\
\hline & Student & 24 & 6 \\
\hline & Employee & 131 & 32.7 \\
\hline & Working & 33 & 8.2 \\
\hline & Free & 71 & 17.8 \\
\hline \multirow{9}{*}{$\begin{array}{l}\text { The way of obtaining health and disease } \\
\text { information }\end{array}$} & Newspapers, periodicals and magazines & 21 & 5.3 \\
\hline & Radio and TV & 190 & 47.5 \\
\hline & IVR & 5 & 1.2 \\
\hline & Internet & 88 & 22 \\
\hline & Doctors and health staff & 68 & 17 \\
\hline & $\begin{array}{l}\text { Booklets, pamphlets, brochures and educational } \\
\text { campaigns }\end{array}$ & 2 & 0.5 \\
\hline & Friends and acquaintances & 12 & 3 \\
\hline & Other & 6 & 1.5 \\
\hline & $\begin{array}{l}\text { Lack of knowledge of the source of health information } \\
\text { and disease }\end{array}$ & 8 & 2 \\
\hline \multirow[t]{4}{*}{ History of hypertension (years) } & $1-5$ & 315 & 78.8 \\
\hline & $6-10$ & 56 & 14 \\
\hline & $11-15$ & 22 & 5.5 \\
\hline & $16-20$ & 7 & 1.7 \\
\hline
\end{tabular}


http://www.ephysician.ir

Table 2. Mean and standard deviation of subscales of health-related quality of life

\begin{tabular}{|l|l|l|l|}
\hline Dimensions and subscales of health-related quality of life & Mean \pm SD) & Minimum Score & Maximum Score \\
\hline Physical health & $54.21( \pm 17.14)$ & 8 & 96 \\
\hline General Health & $50.7( \pm 14.33)$ & 10 & 100 \\
\hline Physical functioning & $70.12( \pm 24.55)$ & 0 & 100 \\
\hline Role limitations due to physical reasons & $62.44( \pm 33.43)$ & 0 & 100 \\
\hline Bodily pain & $52.01( \pm 22.79)$ & 0 & 100 \\
\hline Mental health & $51.68( \pm 17.07)$ & 7 & 94 \\
\hline Role limitations due to emotional reasons & $50.83( \pm 38.62)$ & 0 & 100 \\
\hline Social function & $51.90( \pm 20.29)$ & 0 & 100 \\
\hline Fatigue or vitality & $52.38( \pm 15.84)$ & 5 & 90 \\
\hline mental health & $51.60( \pm 16.16)$ & 4 & 96 \\
\hline General health-related quality of life & $52.94( \pm 15.20)$ & 8 & 90 \\
\hline
\end{tabular}

Table 3. Mean value and SD and levels of health literacy areas on the basis of inadequate, boundary, and adequate

\begin{tabular}{|l|l|l|l|l|l|l|l|}
\hline \multirow{2}{*}{ Variable } & \multirow{2}{*}{$($ Mean \pm SD) } & \multicolumn{2}{|l|}{ Inadequate } & \multicolumn{3}{|l|}{ Boundary } & \multicolumn{2}{l|}{ Adequate } \\
\cline { 3 - 8 } & & $\mathrm{n}$ & $\%$ & $\mathrm{n}$ & $\%$ & $\mathrm{n}$ & $\%$ \\
\hline Health Literacy & $68.66( \pm 13.56)$ & 31 & 7.8 & 220 & 55 & 149 & 37.2 \\
\hline access & $66.56( \pm 16.30)$ & 64 & 16 & 191 & 47.8 & 145 & 36.2 \\
\hline Read & $64.84( \pm 25.15)$ & 113 & 28.3 & 107 & 26.7 & 180 & 45 \\
\hline Understanding & $70.15( \pm 17.39)$ & 61 & 15.3 & 157 & 39.9 & 182 & 45.5 \\
\hline Assessment & $63.67(18.62 \pm)$ & 98 & 24.5 & 172 & 43 & 130 & 32.5 \\
\hline Decision making & $53.07( \pm 12.84)$ & 164 & 41 & 228 & 57 & 8 & 2 \\
\hline
\end{tabular}

Table 4. Correlation between health literacy and health-related quality of life

\begin{tabular}{|l|l|}
\hline Variable & Pearson's correlation coefficient \\
\hline Health Literacy & $\mathrm{r}=0.3$ \\
\hline Health-related quality of life & $\mathrm{p}<0.01$ \\
\hline
\end{tabular}

\section{Discussion}

The results showed that health literacy of patients with hypertension in the study was on the boundary line. This finding is consistent with the results of studies by Tehrani Bani Hashemi et al., Reisi et al., Mollakhalili et al., Motamedi et al., inside our country, and abroad researches by Tung et al. and Moser et al. (8, 22-26). Regarding the findings of the above-mentioned studies, it should be noted that patients with chronic conditions like hypertension are at greater risk of inadequate care and weak consequences, such as lack of knowledge of disease, poor care, increased number of hospitalization and mortality caused by the disease. Thus, patients require adequate health literacy to participate in health care systems and appropriate health decisions (23). In the areas of health literacy, understanding has had the highest average and was at adequate level, this result was consistent with research conducted by Maryam Afshari et al. entitled "Evaluation of health literacy in adults of the city Tuyserkan" (27) and research conducted by Zahedi entitled "The correlation between self-care and health literacy in patients undergoing hemodialysis in Valiasr Hospital in Arak in 2015" (28). In fact, text understanding is a process by which the reader goes through interaction and exposure to written language at the same time means extraction and form. The ability to understand is a mental process that people use to value their written forms and, with this skill, extract meanings of different texts; thus, the cause of obtaining these results is that understanding is mostly under internal factors (28) also the area of decision-making in the field of health literacy have the lowest mean score and was at inadequate and boundary level. Hence, this study was consistent with Mahmoudi and Taheri's study entitled, "The relationship between information literacy and health literacy in graduate students of Ferdowsi University of Mashhad (29) and Zahedi's study (28). It can be said that decision-making needs prerequisite skills such as self-awareness, interpersonal communication, critical thinking, and creative problem-solving are influenced by many factors, including personality, environmental factors, the information, the importance and transparency of the subject, the reward and punishment, correct understanding the situation and values and beliefs. Thus, the decision-making in addition to being influenced by internal factors is affected by the external factors on which the person does not have control or has little control. Thus, it is possible, in spite of a proper understanding of a subject by the person, that an appropriate decision may not be taken (28). If a score of zero to 100 in the questionnaire (SF-36) is set as base. The mean of $50 \pm 10$ can be used as an indicator of community norms and reasonable indicator (30-32). In this study, the 
score of all health-related quality of life correlated with the health of participants in the study was higher than 50; thus, the quality of life in this study can be appropriately evaluated. This result is consistent with results obtained by Nejati, Ghahremani et al., Sabbah et al., Khorsandi et al., Namdar et al. (33-37). Mena-Martin et al. in their study showed that the quality of life in patients with hypertension reduced when the patients are aware of their disease; they also conclude that hypertension alone does not cause a change in quality of life but also labeling that the patient is responsible for it (38). Ahroni and Lawrence et al. in their study, reported repeated reference of people to medical centers, non-specific symptoms of blood pressure and stress as the reasons for decreased quality of life in patients with hypertension compared with healthy subjects (39-40). In this study, the highest score among eight dimensions of health-related quality of life was related to physical function that is consistent with the study conducted by Shamsi et al., entitled "Comparison of the quality of life of military with and without hypertension" (41), and Ebadi's study, entitled "The comparison of the quality of life in men with hypertension and healthy subjects" (42), and is inconsistent with the studies conducted by Namdar et al., entitled "Health-related quality of life in dialysis patients (37), Masroor, entitled "Health-related quality of life in patients with chronic obstructive pulmonary disease (COPD) (43), and the Rahnavard, entitled "Quality of life and its influencing factors in patients with congestive heart failure (CHF) (44).

KockRow and Christensen believe that the prevalence of hypertension is associated with restrictions including changes in diet such as limiting intake of salt and saturated fats, food intake time, weight loss, and changes in everyday tasks (45), but it seems hypertension compared with other chronic diseases such as COPD and CHF, which reduces cardiac output, increased energy for breathing and insomnia caused by respiratory distress and cough (46) less affects physical function. However, in this study, physical health subscale obtained higher score than the mental health subscale that this issue was consistent with the results of Hadi et al. (47) and Khorsandi et al. (36). It seems that this is due to the mental aspects of mental health and greater influence of individual assessment of on it. Rejeski et al. found that physical activity can improve health-related quality of life (48). It is recommended that individuals, especially those with high blood pressure, engage in more physical activity (47). The results of this study also showed a significant positive correlation between health literacy and health-related quality of life. These findings in domestic studies are consistent with the results obtained by Kooshyar et al., Hosseini et al., and in foreign studies are consistent with Macabasco-O'Connell et al. and Song et al. (52-49). In contrast with the results obtained by Zhang et al., health-related quality of life health literacy in patients with rheumatoid had no effect (53). The difference of Zhang's results with the results of this study may be related to too the rapid estimate of adult literacy in medicine (REALM). The use of this tool is to evaluate the ability of patients to understand that the patient's functional health literacy is still open for discussion and doubts (49); on the other hand, the culture and ethnicity of people are the factors affecting health and health literacy of people (55-54) as the family, cultural, and social impacts have been critical in shaping people's attitudes and beliefs and affects how people interact with the health system (56). Therefore, one of the main causes of these differences could be cultural differences.

\section{Conclusions}

In summary, the findings of this study showed a significant positive correlation between health literacy and healthrelated quality of life. Thus, more attention in the identification of patients with hypertension and improving health literacy of patients through approaches such as the information simplification, the use of simple and understandable educational materials, and taking advantage of experts to plan and design health education programs, we can take steps to improve the quality of life in patients with hypertension.

\section{Acknowledgments:}

This study is the result of the nursing master's degree thesis approved in 02/27/2016 in Shahid Beheshti University of Medical Sciences in Tehran. Therefore, the graduate office of the university and sincere cooperation of the management and personnel of hospitals and nursing that this research would not be possible without the cooperation of them are admired.

\section{Conflict of Interest:}

There is no conflict of interest to be declared.

Authors' contributions:

All authors contributed to this project and article equally. All authors read and approved the final manuscript. 


\section{References:}

1) Naji A, Naroie S, Abdeyazdan G, Dadkani E. Effect of Applying Self-Care Orem Model on Quality of Life in the Patient under Hemodialysis. Zahedan Journal of Research in Medical Sciences 2012; 14 (1): 8-12.

2) Ahmadi A, Hasanzadeh J, Rajaefard A. To Determine the Relative Factors on Hypertension in Kohrang, Chaharmahal \& Bakhtiari Province, 2007. IRJE journal. 2008; 4 (2): 19-25.

3) Nasri H. On the Occasion of World Hypertension Day 2016. J Isfahan Med Sch 2016; 34 (374): 229-31.

4) Zadirad H, Masoudi GR, Zareban I, Shahraki Poor M, Jadgal K. The Effect of Educational Program Based on BASNEF Model on Women's Blood Pressure with Hypertension. Journal of Torbat Heydariyeh University of Medical Sciences. 2013; 1 (2): 22-31.

5) Kandula NR, Nsiah-Kumi PA, Makoul G, Sager J, Zei CP, Glass S, et al. The relationship between health literacy and knowledge improvement after a multimedia type 2 diabetes education program. Patient education and counseling. 2009; 75 (3): 321-327. doi: 10.1016/j.pec.2009.04.001. PMID: 19395223.

6) Sorensen K, Van den B roucke S, Fullam J, Doyle G, Pelikan J, Slonska Z, et al. Health literacy and pubilc health: a systematic review and integration of definitions and models. Bio Ma Center Public Health. 2012; 12 (80): 1-13. doi: 10.1186/1471-2458-12-80. PMID: 22276600, PMCID: PMC3292515.

7) Kickbusch I, Pelikan JM, Apfel F, Tsouros A. Health Literacy The Solid Facts. Copenhagen: World Health Organization (WHO); 2013.

8) Tehrani Bani hashemi S, Amirkhani MA, Haghdoost AA, Alavian S, Asgharifard H, Baradaran H, et al. Health Literacy and the Influencing Factors: A Study in Five Provinces of Iran. Strides Dev Med Educ. 2007; 4 (1) :1-9.

9) Jabalameli S, Ther Neshat doost H, Moulavi H. Efficacy of cognitive-behavioral stress management intervention on quality of life and blood pressure in female patients with hypertension. SJKU. 2010; 15 (2): 88-97.

10) Niu SE, Li IC. Quality of life of patients having renal replacement therap. Journal of Advanced Nursing. 2005; 51 (1): 15-21. doi: 10.1111/j.1365-2648.2005.03455.x.

11) Cote 1, Farris K, Feeny D. Is adherence to drug treatment correlated with health-related quality of life? Quality of life research. 2003; 12 (6): 621-633. doi: 10.1023/A:1025180524614.

12) Shabany Hamedan M, Mohammad AJ, Shekarabi R, Hosseini A. The Relationship Between Medication Adherence and Quality of Life in Renal Transplant Patients. IJN. 2011; 23 (67): 29-34.

13) Lin xJ, Lin IM and Fan SY. Methodological issues in Measuring health-related quality of life. Tzu Chi Medical Journal. 2012; 25 (1): 8-12. doi: 10.1016/j.tcmj.2012.09.002

14) Mcnaughton CD, Jacobson TA, Kripalani S. Low literacy is associated with uncontrolled blood pressure in primary care patients with hypertension and heart disease. Patient education and counseling. 2014; 96(2): 165-170. doi: 10.1016/j.pec.2014.05.007

15) Rothman RL, et al. Influence of patient literacy on the effectiveness of a primary care-based diabetes disease management program. Jama. 2004; 292 (14): 1711-1716. doi:10.1001/jama.292.14.1711

16) Willens DE, et al. Association of brief health literacy screening and blood pressure in primary care. Journal of health communication, 2013; 18 (1): 129-142. doi: 10.1080/10810730.2013.825663. PMID: 24093351, PMCID: PMC3815081.

17) Powers BJ, Olsen MK, Oddone EZ, Thorpe CT, Bosworth HB. Literacy and blood pressure-do healthcare systems influence this relationship? A cross-sectional study. BMC health services research. 2008; 8 (1): 219. doi: 10.1186/1472-6963-8-219. PMID: 18947408, PMCID: PMC2600788.

18) War JE, Sherbourne CD. The MOS 36-item short form health survey (SF-36). I. Conceptual framework and item selection. Med Care 1992; 30 (6): 473-83. PMID: 1593914.

19) Montazeri A, Goshtasbi A, Vahdaninia MAS. The short form health survey (SF-36): Translation and validation study of the Iranian version. Payesh 2006; 5 (1): 49-56.

20) Dehdari T, Heydarnia A, Ramezankhani A, Sadeghian S, Ghofrani Pour Rouchi FA, Babaei GH, et al. Effects of phase III cardiac rehabilitation programs on anxiety and quality of life in anxious patients after coronary artery bypass surgery. The Journal of Tehran University Heart Center 2007; 2 (7): 207-212.

21) Montazeri A, Tavousi M, Rakhshani F, Azin SA, Jahangiri K, Ebadi M, et al. Health Literacy for Iranian Adults (HELIA): develoment and psychometric properties. Payesh. 2014; 13 (5): 589-599.

22) Reisi M, Javadzade H, Mostafavi F, Tavassoli E, Sharifirad Gh. Health Literacy and Health Promoting Behaviors among OlderAdults. J Health Syst Res. 2013; 9 (8): 827-836.

23) Mollakhalili H, Papi A, Sharifirad Gh, Zare-Farashbandi F, Hasanzadeh A. Health literacy in patients hospitalized at educational hospitals affi liated to Isfahan University of Medical Sciences. Health information management. 2014; 11 (4): 364-373. 
24) Motamedi F, Malekzadeh S, Azami M, Mirzaei M. Health literacy of cardiovascular patients admitted to private hospitals in Kerman. International Journal of Advanced Biotechnology and Research. 2016; 7 (1): 239-247.

25) Tung HH, Lu TM, Chen LK, Liang Sh, Wu SF, Chu KH. Health literacy impact on elderly patients with heart failure in Taiwan. Journal of Clinical Gerontology \& Geriatrics. 2014; 5 (3): 72-76. doi: 10.1016/j.jcgg.2014.01.005

26) Moser DK, Robinson S, Biddle MJ, Pelter MM, Nesbitt TS, Suthard J, et al. Health literacy predicts morbidity and mortality in rural patients with heart failure. Journal of Cardiac Failure. 2015; 21 (8): 612618. doi: 10.1016/j.cardfail.2015.04.004

27) Afshari M, Khazaei S, Bahrami M, Merati H. Investigating Adult Health Literacy in Tuyserkan City. Journal of Education and Community Health. 2014; 1 (2): 48-55. doi: 10.20286/jech-010248

28) Zahedi S. The correlation between self-care and health literacy in patients undergoing hemodialysis in Valiasr Hospital in Arak in 2015 [dissertation]. Shahid Beheshti University of Medical Sciences and Health; 2016.

29) Mahmoudi H, Taheri A. Relation between Information Literacy and Health Literacy of Students in Ferdowsi University of Mashhad. HII. 2015; 2 (2): 31-41.

30) Ahmadi F, Salar A, Faghihzadeh S. Quality of life in Zahedan elderly population. Hayat. $2004 ; 10$ (3): 61 67.

31) Ali-Akbari F, Khalifehzadeh A, Parvin N. The effect of short time telephone follow-up on physicalconditions and quality of life in patients after pacemaker implantation. J Shahrekord Med Sci. 2009; 11 (3): 23-28.

32) Nejati V, Ashayeri H. Health related quality of life in the elderly in Kashan. Iranian J Psychiatr Clin Psychol. 2008; 14(1): 56-61.

33) Nejati V, Ashayeri H. Health Related Quality of Life in the Elderly in Kashan. IJPCP. $2008 ; 14$ (1): $56-61$.

34) Ghahremani L, Nazari M, Mosavi M. Improvement of Quality of Life in Elderly men in Kahrizak nursing home based on educational intervention. Knowledge \& Health 2008;4 (2): 18-23.

35) Sabbah I, Drouby N, Sabbah S, Retel-Rude N, Mercier M. Quality of life in rural and urban populations in Lebanon using SF-36 Health Survey. Health and Quality of Life Outcomes. 2003; 1 (30): 1-14. Doi: 10.1186/1477-7525-1-30

36) Khorsandi M, Jahani F, Rafie M, Farazi A. Health-related quality of life in staff and hospital personnel of Arak university of medical sciences in 2009. Arak Med Univ J. 2010; 13 (1): 40-48.

37) Namdar A, Beigizadeh S, Najafipour S. Health- related quality of life in dialysis patients. Journal of Jahrom University of Medical Sciences. 2013; 10 (4): 19-27.

38) Mena-Martin FJ, Martin-Escudero JC, Simal-Blanco F, Carretero-Ares JL, Arzua-Mouronte D, HerrerosFernandez V. Health-related quality of life of subjects with known and unknown hypertension: results from the population-based Hortega study. Journal of Hypertension. 2003; 21 (7): 1283-1289. doi: 10.1097/01.hjh.0000059086.43904.17. PMID: 12817174.

39) Ahroni JH, Boyko EJ. Responsiveness of the SF-36 among veterans with diabetes mellitus. J Diabet Complications. 2000; 14 (1): 31-9. PMID: 10925064. doi: 10.1016/S1056-8727(00)00066-0

40) Lawrence WF, Fryback DG, Martin PA, Klein R, Klein BE. Health status and hypertension: apopulationbased study. J Clin Epidemiol. 2004; 49 (11): 1239-45. PMID: 8892491. doi: 10.1016/S08954356(96)00220-X

41) Shamsi A, akbarRefahi A, Malmir M, Mollahadi M, Ebadi A. Comparison of quality of life in military personnel with and without hypertension. Ann Mil Health Sci Res. 2012; 10 (3): 213-218.

42) Ebadi A, Shamsi A, Refahi AA, Saied Y. Comparison of the Quality of Life in Men with and Without Hypertension. Sci J Hamdan Nurs Midwifery Fac. 2012; 20 (1): 5-13.

43) Masroor DD, Rafii F, Fadaeeaghdam N, Hoseini AF. Health-Related Quality of Life in Patients with Chronic Obstructive Pulmonary Disease. IJN. 2012; 25 (76): 19-27.

44) Rahnavard Z, Zolfaghari M, Kazemnejad A, Hatamipour K. An investigation of quality of life and factors affecting it in the patients with congestive heart failure. Hayat. 2006; 12 (1): 77-86.

45) Christensen BI, KockRow ED. Adult health nursing. St. Louis: Mosby; 1999.

46) Smeltzer SC, Bare BG. Textbook of medical surgical nursing. Philadelphia: Lippincott Williams \& Wilkins; 2004.

47) Hadi N, Malekan Makan L. Evaluation of health state and quality of life in primary school teachers of Shiraz, 2003. Med J Hormozgan Univ. 2007; 10 (4): 387-392. 
48) Rejeski WJ, Mihalko SL. Physical activity and quality of life in older adults. J Gerontol Series A Biol Sci Med Sci. 2001; 56 (2): 23-35. doi: 10.1093/gerona/56.suppl_2.23

49) Kooshyar H, Shoorvazi M, Dalir Z, Hosseini M. Health Literacy and its Relationship with Medical Adherence and Health-Related Quality of Life in Diabetic Community-Residing Elderly. J Mazandaran Univ Med Sci. 2014; 23 (1): 134-143.

50) Hosieni F, Mirzaei T, Ravari A, Akbary A. The relationship between health literacy and quality of life in retirement of Rafsanjan University of Medical Sciences. Journal of Health Literacy. 2016; 1 (2): 92-99.

51) Macabasco-O' Connell A, De Walt DA, Broucksou KA, Hawk V, Baker DW, Schillinger D, et al. Relationship between literacy, knowledge, self-care behaviors, and heart failure-related quality of life among patients with heart failure. J Gen Intern Med. 2011; 26 (9): 979-986. doi: 10.1007/s11606-0111668-y. PMID: 21369770, PMCID: PMC3157534.

52) Song L, Mishel M, Bensen JT, Chen RC, Knafl GJ, Blackard B, et al. How does health literacy affect quality of life among men with newly diagnosed clinicallylocalized prostate cancer. Cancer. 2012; 118 (15): 3842-3851. doi: 10.1002/cncr.26713. PMID: 22180041.

53) Zhang XH, Li SC, Fong KY, Thumboo J. The impact of health literacy on healthrelated quality of life (HRQoL) and utility assessment among patients with rheumatic diseases.Value Health. 2009; 12 Suppl 3:S 106-109. doi: 10.1111/j.1524-4733.2009.00640.x. PMID: 20586970.

54) Osborn CY, Cavanaugh K, Wallston KA, Kripalani S, Elasy TA, Rothman RL, et al. Health literacy explainsracial disparities in diabetes medication adherence. J Health Commun. 2011; 16 Suppl 3: 268-278. doi: 10.1080/10810730.2011.604388. PMID: 21951257, PMCID: PMC3561717.

55) Shaw SJ, Huebner C, Armin J, Orzech K, Vivian J. The role of culture in health literacy and chronic disease screening and management. J Immigr Minor Health. 2009; 11 (6): 460-467. doi: 10.1007/s10903008-9135-5. PMID: 18379877.

56) Von Wagner C, Knight K, Steptoe A, Wardle J. Functional health literacy and health promoting behaviour in a national sample of British adults. J EpidemiolCommunity Health. 2007; 61 (12): 1086-1090. doi: 10.1136/jech.2006.053967. PMID: 18000132, PMCID: PMC2465677. 